\title{
Computing multivariate Fekete and Leja points by numerical linear algebra*
}

\author{
L. Bos ${ }^{1}$, S. De Marchi ${ }^{2}$, A.Sommariva ${ }^{2}$ and M.Vianello ${ }^{2}$
}

July 26, 2010

\begin{abstract}
We discuss and compare two greedy algorithms, that compute discrete versions of Fekete-like points for multivariate compact sets by basic tools of numerical linear algebra. The first gives the so-called "Approximate Fekete Points" by QR factorization with column pivoting of Vandermonde-like matrices. The second computes Discrete Leja Points by LU factorization with row pivoting. Moreover, we study the asymptotic distribution of such points when they are extracted from Weakly Admissible Meshes.
\end{abstract}

2000 AMS subject classification: 41A10, 41A63, 65D05.

Keywords: Weakly Admissible Meshes, Approximate Fekete Points, Discrete Leja Points, Vandermonde matrices, QR factorization with column pivoting, LU factorization with row pivoting, pluripotential theory, equilibrium measure.

\section{Introduction.}

Locating good points for multivariate polynomial approximation, in particular polynomial interpolation, is an open challenging problem, even in standard domains. One set of points that is always good, in theory, are the so-called Fekete points. They are defined to be those points that maximize the (absolute value of the) Vandermonde determinant on the given compact set. However, these are known analytically in only a few instances (the interval and the complex circle for univariate interpolation, the cube for

\footnotetext{
*Supported by the "ex-60\%" funds and by the project "Interpolation and Extrapolation: new algorithms and applications" of the University of Padova, and by the INdAM GNCS.

${ }^{1}$ Dept. of Computer Science, University of Verona, Italy e-mail: leonardpeter.bos@univr.it

${ }^{2}$ Dept. of Pure and Applied Mathematics, University of Padova, Italy e-mail: demarchi, alvise, marcov@math.unipd.it
} 
tensor product interpolation), and are very difficult to compute, requiring an expensive and numerically challenging multivariate optimization.

Recently, a new insight has been given by the theory of "admissible meshes" of Calvi and Levenberg [15], which are nearly optimal for leastsquares approximation and contain interpolation sets nearly as good as Fekete points of the domain. These allow us to replace a continuous compact set by a discrete version, that is "just as good" for all practical purposes.

In some recent papers, a simple and effective greedy algorithm to extract approximate Fekete points from admissible meshes has been studied, and succesfully applied in various instances, cf. [8, 9, 34, 35]. The algorithm gives an approximate solution to a nonlinear combinatorial optimization problem (discrete maximization of the Vandermonde determinant) using only a basic tool of numerical linear algebra, namely the QR factorization with column pivoting.

In this paper, we pursue an alternative greedy algorithm for discrete maximization on (weakly) admissible meshes, i.e., the computation of the so-called Leja points of the mesh. Following an idea recently proposed by $\mathrm{R}$. Schaback [32], we show that this algorithm can be easily implemented by another basic tool of linear algebra, the LU factorization with partial (row) pivoting. We recall that approximate Fekete points, computed with any basis from a weakly admissible mesh, are asymptotically equidistributed with respect to the pluripotential-theoretic equilibrium measure of the compact set; cf. [8]. Here we prove that the same is true for discrete Leja points computed with a special class of polynomial bases.

\section{Some definitions and notation.}

Suppose that $X \subset \mathbb{C}^{d}$ is a compact set. Let $\mathbb{P}_{n}^{d}(X)$ denote the space of polynomials (on $\mathbb{C}^{d}$ ) of degree at most $n$, restricted to $X$. We will assume that $X$ is $\mathbb{P}_{n}^{d}$-determining, i.e., if $p \in \mathbb{P}_{n}^{d}$ is identically zero on $X$, then it is identically zero on $\mathbb{C}^{d}$.

The following matrix and its determinant will be important. For any ordered set of points $\mathbf{z}=\left\{z_{1}, z_{2}, \ldots, z_{k}\right\} \in \mathbb{C}^{d}$ and ordered set of polynomials $\mathbf{q}=\left\{q_{1}, q_{2}, \ldots, q_{m}\right\}$ on $\mathbb{C}^{d}$, let

$$
V(\boldsymbol{z} ; \boldsymbol{q})=V\left(z_{1}, \ldots, z_{k} ; q_{1}, \ldots, q_{m}\right):=\left[q_{j}\left(z_{i}\right)\right]_{1 \leq i \leq k, 1 \leq j \leq m} \in \mathbb{C}^{k \times m}
$$

denote the associated Vandermonde matrix. The ordering of the sets $\mathbf{z}$ and $\mathbf{q}$ are important, giving the ordering of the rows and columns, respectively of the matrix. It is especially important to note that the rows of this matrix correspond to the points $z_{i}$ and the columns to the polynomials $q_{j}$. In particular, selecting a row in the matrix corresponds to selecting a point in the set $\mathbf{z}$. 
In the case when $m=k$ we let

$$
v d m(\mathbf{z} ; \mathbf{q})=v d m\left(z_{1}, \ldots, z_{k} ; q_{1}, \ldots, q_{k}\right):=\operatorname{det}\left(V\left(z_{1}, \ldots, z_{k} ; q_{1}, \ldots, q_{k}\right)\right)
$$

denote the determinant of the Vandermonde matrix.

In the following, we adopt the notation:

$$
\|f\|_{X}:=\sup _{x \in X}|f(x)|
$$

where $f$ is any bounded function on the set $X$.

\section{Fekete points.}

The concept of Fekete points for interpolation can be described in a very general, not necessarily polynomial, setting. It is worth observing that such Fekete points should not be confused with the "minimum energy" Fekete points (cf., e.g., [2] and references therein), the two concepts being equivalent only in the univariate complex case (cf., e.g., [27]).

Given a compact set $K \subset \mathbb{C}^{d}$, a finite-dimensional space of linearly independent continuous functions,

$$
S_{N}=\operatorname{span}\left(p_{j}\right)_{1 \leq j \leq N},
$$

and a finite set $\left\{\xi_{1}, \ldots, \xi_{N}\right\} \subset K$, if

$$
v d m\left(\xi_{1}, \ldots, \xi_{N} ; p_{1}, \ldots, p_{N}\right) \neq 0
$$

then the set $\left\{\xi_{1}, \ldots, \xi_{N}\right\}$ is unisolvent for interpolation in $S_{N}$, and

$$
\ell_{j}(x)=\frac{v d m\left(\xi_{1}, \ldots, \xi_{j-1}, x, \xi_{j+1}, \ldots, \xi_{N} ; \boldsymbol{p}\right)}{v d m\left(\xi_{1}, \ldots, \xi_{j-1}, \xi_{j}, \xi_{j+1}, \ldots, \xi_{N} ; \boldsymbol{p}\right)}, j=1, \ldots, N,
$$

is a cardinal basis, i.e. $\ell_{j}\left(\xi_{k}\right)=\delta_{j k}$ and

$$
L_{S_{N}} f(x)=\sum_{j=1}^{N} f\left(\xi_{j}\right) \ell_{j}(x)
$$

interpolates any function $f$ at $\left\{\xi_{1}, \ldots, \xi_{N}\right\}$. In matrix terms, the cardinal basis $\boldsymbol{\ell}=\left(\ell_{1}, \ldots, \ell_{N}\right)$ is obtained from the original basis $\boldsymbol{p}=\left(p_{1}, \ldots, p_{N}\right)$ as

$$
\boldsymbol{\ell}^{t}=L \boldsymbol{p}^{t}, \quad L:=\left((V(\boldsymbol{\xi} ; \boldsymbol{p}))^{-1}\right)^{t} .
$$

In the case that such points maximize the (absolute value of the) denominator of (4) in $K^{N}$ (Fekete points), then $\left\|\ell_{j}\right\|_{\infty} \leq 1$ for every $j$, and 
thus the norm of the interpolation operator $L_{S_{N}}: C(K) \rightarrow S_{N}$ is bounded by the dimension of the interpolation space,

$$
\left\|L_{S_{N}}\right\|=\max _{x \in K} \sum_{j=1}^{N}\left|\ell_{j}(x)\right|=\max _{x \in K}\left\|L \boldsymbol{p}^{t}(x)\right\|_{1} \leq N .
$$

Clearly, Fekete points as well as $\left\|L_{S_{N}}\right\|$ are independent of the choice of the basis in $S_{N}$, since the determinant of the Vandermonde-like matrices changes by a factor independent of the points (namely the determinant of the transformation matrix between the bases).

In the present polynomial framework, we have that

$$
S_{N}=\mathbb{P}_{n}^{d}(K), \quad N=\operatorname{dim}\left(\mathbb{P}_{n}^{d}(K)\right)
$$

and that

$$
\Lambda_{n}:=\left\|L_{S_{N}}\right\|
$$

is the so-called Lebesgue constant of interpolation at the point set $\left\{\xi_{j}\right\}, K$ being any $\mathbb{P}_{n}^{d}$-determining compact set. In this framework Fekete points and Lebesgue constants are preserved under affine mapping of the domain. It is also worth recalling that (7) is often a rather pessimistic overestimate of the actual growth.

There are several open problems about Fekete points, whose properties have been studied till now mainly in the univariate complex case in view of their deep connection with potential theory. They are analytically known only in few cases: the interval (Gauss-Lobatto points) where $\Lambda_{n}=\mathcal{O}(\log n)$, the complex circle (equispaced points) where again $\Lambda_{n}=\mathcal{O}(\log n)$, and the cube (tensor-product of Gauss-Lobatto points) for tensorial interpolation where $\Lambda_{n}=\mathcal{O}\left(\log ^{d} n\right)$, cf. [12]. An important qualitative result has been proved only recently, namely that Fekete points are asymptotically equidistributed with respect the pluripotential equilibrium measure of $K$, cf. [3]. Their asymptotic spacing is known only in few instances, cf. the recent paper [10].

Moreover, the numerical computation of Fekete points becomes rapidly a very large scale problem, namely a nonlinear optimization problem in $N \times d$ variables. It has been solved numerically only in very special cases, like the triangle (up to degree $n=19$, cf. [36]) and the sphere (up to degree $n=191$, cf. [33]).

A reasonable approach for the computation of Fekete points is to use a discretization of the domain, moving from the continuum to nonlinear combinatorial optimization. But which could be a suitable starting mesh? A possible answer is given by the theory of admissible meshes for multivariate polynomial approximation, recently studied by Calvi and Levenberg [15], which is briefly sketched in the following section. 


\section{Weakly Admissible Meshes (WAMs).}

Given a polynomial determining compact set $K \subset \mathbb{C}^{d}$, we define a Weakly Admissible Mesh (WAM) to be a sequence of discrete subsets $\mathcal{A}_{n} \subset K$ such that

$$
\|p\|_{K} \leq C\left(\mathcal{A}_{n}\right)\|p\|_{\mathcal{A}_{n}}, \quad \forall p \in \mathbb{P}_{n}^{d}(K)
$$

where both $\operatorname{card}\left(\mathcal{A}_{n}\right) \geq N$ and $C\left(\mathcal{A}_{n}\right)$ grow at most polynomially with $n$. When $C\left(\mathcal{A}_{n}\right)$ is bounded we speak of an Admissible Mesh (AM).

We sketch below the main features of WAMs in terms of ten properties (cf. $[8,15])$ :

P1: $C\left(\mathcal{A}_{n}\right)$ is invariant under affine mapping

P2: any sequence of unisolvent interpolation sets whose Lebesgue constant grows at most polynomially with $n$ is a WAM, $C\left(\mathcal{A}_{n}\right)$ being the Lebesgue constant itself

P3: any sequence of supersets of a WAM whose cardinalities grow polynomially with $n$ is a WAM with the same constant $C\left(\mathcal{A}_{n}\right)$

P4: a finite union of WAMs is a WAM for the corresponding union of compacts, $C\left(\mathcal{A}_{n}\right)$ being the maximum of the corresponding constants

P5: a finite cartesian product of WAMs is a WAM for the corresponding product of compacts, $C\left(\mathcal{A}_{n}\right)$ being the product of the corresponding constants

P6: in $\mathbb{C}^{d}$ a WAM of the boundary $\partial K$ is a WAM of $K$ (by the maximum principle)

P7: given a polynomial mapping $\pi_{s}$ of degree $s$, then $\pi_{s}\left(\mathcal{A}_{n s}\right)$ is a WAM for $\pi_{s}(K)$ with constants $C\left(\mathcal{A}_{n s}\right)$ (cf. [8, Prop.2])

P8: any $K$ satisfying a Markov polynomial inequality like $\|\nabla p\|_{K} \leq M n^{r}\|p\|_{K}$ has an AM with $\mathcal{O}\left(n^{r d}\right)$ points (cf. [15, Thm.5])

P9: least-squares polynomial approximation of $f \in C(K)$ (cf. [15, Thm.1]): the least-squares polynomial $\mathcal{L}_{\mathcal{A}_{n}} f$ on a WAM is such that

$$
\left\|f-\mathcal{L}_{\mathcal{A}_{n}} f\right\|_{K} \lesssim C\left(\mathcal{A}_{n}\right) \sqrt{\operatorname{card}\left(\mathcal{A}_{n}\right)} \min \left\{\|f-p\|_{K}, p \in \mathbb{P}_{n}^{d}(K)\right\}
$$

P10: Fekete points: the Lebesgue constant of Fekete points extracted from a WAM can be bounded like $\Lambda_{n} \leq N C\left(\mathcal{A}_{n}\right)$ (that is the elementary classical bound of the continuum Fekete points times a factor $\left.C\left(\mathcal{A}_{n}\right)\right)$; moreover, their asymptotic distibution is the same of the continuum Fekete points, in the sense that the corresponding discrete probability measures converge weak- $*$ to the pluripotential equilibrium measure of $K$ (cf. [8, Thm.1]) 
The properties above give the basic tools for the construction and application of WAMs in the framework of polynomial interpolation and approximation. For illustrative purposes we focus briefly on the real bivariate case, i.e. $K \subset \mathbb{R}^{2}$. Property P8, applied for example to convex compacts where a Markov inequality with exponent $r=2$ always holds, says that it is always possible to obtain an Admissible Mesh with $\mathcal{O}\left(n^{4}\right)$ points. In order to avoid such a large cardinality, which has severe computational drawbacks, we can turn to WAMs, which can have a much lower cardinality, typically $\mathcal{O}\left(n^{2}\right)$ points.

In [8] a WAM on the disk with about $2 n^{2}$ points and $C\left(\mathcal{A}_{n}\right)=\mathcal{O}\left(\log ^{2} n\right)$ has been constructed with standard polar coordinates, using essentially property P2 for univariate Chebyshev and trigonometric interpolation. Moreover, using property $\mathrm{P} 2$ and $\mathrm{P} 7$, WAMs for the triangle and for linear trapezoids, again with about $2 n^{2}$ points and $C\left(\mathcal{A}_{n}\right)=\mathcal{O}\left(\log ^{2} n\right)$, have been obtained simply by mapping the so-called Padua points of degree $2 n$ from the square with standard quadratic transformations. We recall that the Padua points are the first known optimal points for bivariate polynomial interpolation, with a Lebesgue constant growing like log-squared of the degree (cf. $[7,14])$.

In [11] these results have been improved, showing that there are WAMs for the disk and for the triangle with approximately $n^{2}$ points and still the same constants. Property P4 allows to obtain WAMs for any polygon that can be subdivided into triangles or trapezoids by standard algorithms of computational geometry.

\section{Approximate Fekete points.}

The WAMs described in the previous section can be used directly for leastsquares approximation of continuous functions, by property P10. On the other hand, it is also important to identify good interpolation points. Once we have a WAM, by property P10 we can try to compute Fekete points of the WAM, that is to solve the nonlinear discrete optimization problem. The latter, however, is known to be NP-hard [16], so heuristic or stochastic algorithms are mandatory.

To this purpose, we can adopt the greedy algorithm recently studied in $[8,9,34]$, which gives an approximate solution using only optimized tools of numerical linear algebra (namely QR-like factorizations). Consider a WAM $\left\{\mathcal{A}_{n}\right\}$ of a polynomial determining compact set $K \subset \mathbb{C}^{d}$

$$
\boldsymbol{a}=\mathcal{A}_{n}=\left\{a_{1}, \ldots, a_{M}\right\}, \quad M \geq N=\operatorname{dim}\left(\mathbb{P}_{n}^{d}(K)\right)
$$

and the associated rectangular Vandermonde-like matrix

$$
V(\boldsymbol{a} ; \boldsymbol{p})=V\left(a_{1}, \ldots, a_{M} ; \boldsymbol{p}\right):=\left[p_{j}\left(a_{i}\right)\right], 1 \leq i \leq M, 1 \leq j \leq N
$$


where $\left\{p_{j}\right\}$ is a basis of $\mathbb{P}_{n}^{d}(K)$. We sketch below the algorithm in a Matlablike notation.

\section{Algorithm AFP: Approximate Fekete Points}

(i) $V_{0}=V(\boldsymbol{a} ; \boldsymbol{p}) ; T_{0}=I ;($ inizialization)

for $k=0, \ldots, s-1$ (successive orthogonalization)

$$
\begin{aligned}
& V_{k}=Q_{k} R_{k} ; P_{k}=\operatorname{inv}\left(R_{k}\right) ; \\
& V_{k+1}=V_{k} P_{k} ; T_{k+1}=T_{k} P_{k} ;
\end{aligned}
$$

end ;

(ii) ind $=[] ; W=V_{s}^{t}$; (inizialization)

for $k=1, \ldots, N$ (greedy algorithm for a maximum volume submatrix)

- "select the largest norm column $\operatorname{col}_{i_{k}}(W)$ "; ind $=\left[\right.$ ind, $\left.i_{k}\right]$;

- "remove from every column of $W$ its orthogonal projection onto $\operatorname{col}_{i_{k}} "$

end ;

(iii) $\boldsymbol{\xi}=\boldsymbol{a}($ ind $)$; (extraction of Approximate Fekete Points)

The core of the algorithm is given by $(i i)$ and can be efficiently implemented by the well-known $Q R$ factorization with column pivoting [13]. In Matlablike programming, the greedy algorithm simply reduces to the following instructions

$$
\boldsymbol{w}=W \backslash \boldsymbol{b} ; \text { ind }=\text { find }(\boldsymbol{w} \neq \mathbf{0}) ;
$$

where $\boldsymbol{b}$ is any nonzero column vector, since the "backslash" standard solver uses exactly the QR factorization with column pivoting when applied to an undetermined system. The purpose of $(i)$ is to manage the ill-conditioning that arises with nonorthogonal bases, like the standard monomial basis. Indeed, it eventually amounts to a change of basis from $\boldsymbol{p}=\left(p_{1}, \ldots, p_{N}\right)$ to the discrete orthonormal basis

$$
\boldsymbol{\varphi}=\left(\varphi_{1}, \ldots, \varphi_{N}\right)=\boldsymbol{p} T_{s}
$$

with respect to the inner product

$$
\langle f, g\rangle=\sum_{i=1}^{M} f\left(a_{i}\right) \overline{g\left(a_{i}\right)} .
$$

In practice $s=1$ or 2 iterations suffice, unless the original matrix $V(\boldsymbol{a} ; \boldsymbol{p})$ is so severely ill-conditioned (rule of thumb: condition number much greater 
than the reciprocal of machine precision) that the algorithm fails. This well-known phenomenon of "twice is enough" in numerical Gram-Schmidt orthogonalization, has been deeply studied and explained in [21]. Observe that we don't really need orthogonality, but rather a reasonable conditioning of the Vandermonde matrix in the transformed basis, namely $V(\boldsymbol{a} ; \boldsymbol{\varphi})=$ $V(\boldsymbol{a} ; \boldsymbol{p}) T_{s}=V_{s}$.

The quality of the approximate Fekete points given by Algorithm AFP is in general affected by the choice of the basis. Nevertheless, it has been proved in $[8$, Thm.1] that their asymptotic distribution is the same of true Fekete points, in the sense that the corresponding discrete probability measures converge weak-* to the pluripotential equilibrium measure of $K$.

\section{Leja points.}

Let $X \subset \mathbb{C}^{d}$ be a continuous or discrete compact set. Suppose again that we are given an ordered basis

$$
\boldsymbol{p}=\left\{p_{1}, \ldots, p_{N}\right\}
$$

for $\mathbb{P}_{n}^{d}(X)$. Here, of course, $N:=\operatorname{dim}\left(\mathbb{P}_{n}^{d}(X)\right)$. The Leja points for $X$, up to degree $n$, with respect to the ordered basis $\boldsymbol{p}$, is a sequence of points $\xi_{1}, \xi_{2}, \ldots, \xi_{N} \in X$, defined as follows.

The first point, $\xi_{1}$, is defined to the max point of $\left|p_{1}\right|$ on $X$ (if there is more than one then each choice will lead to a different Leja sequence). Suppose then that the points $\xi_{1}, \xi_{2}, \ldots, \xi_{k}$ have already been chosen. The next point $\xi_{k+1} \in X$ is chosen to be a max point of the function

$$
x \mapsto\left|v d m\left(\xi_{1}, \ldots, \xi_{k}, x ; p_{1}, \ldots, p_{k}, p_{k+1}\right)\right| .
$$

Again, if there is more than one max point, each choice leads to a different sequence. This procedure can be seen as a greedy algorithm for the maximization of the Vandermonde determinant in the set $X$.

There are also "non determinantal" versions of multivariate Leja points, which are related to potential theory and minimum energy configurations (the two concepts being equivalent in the univariate complex setting). For an overview about theoretical and computational aspects of Leja points we may quote, e.g., $[1,4,5,17,23,28,31]$ and references therein.

\subsection{The LU decomposition and partial pivoting.}

Suppose that $k \geq m$. If Gaussian elimination can be, and is, directly applied to the matrix $A \in \mathbb{C}^{k \times m}$ the end result is the so-called LU decomposition $A=L U$ where $L \in \mathbb{C}^{k \times m}$ is lower triangular with $L_{j j}=1, j=1, \ldots, m$, 
and $U \in \mathbb{C}^{m \times m}$ is upper triangular. This, as it turns out, is not always possible, as is easily verified, for example, for the matrix

$$
A=\left[\begin{array}{ll}
0 & 1 \\
1 & 0
\end{array}\right]
$$

If the rows of $A$ are allowed to be permuted then an LU decompostion of the form $P A=L U$, where $P \in \mathbb{C}^{k \times k}$ is a certain permutation matrix, is always possible. The simplest such strategy for permuting the rows is called partial (or row) pivoting and it works as follows.

Gaussian elimination is the process of sequentially subtracting from certain rows a multiple of the "pivot row" with the purpose of producing an equivalent matrix with as many zeros in it as possible. Partial pivoting is a simple strategy for choosing the pivot rows. The first pivot row is that row $i=p_{1}$ so that the element $\left|A_{p_{1} 1}\right|$ is as large as possible. Then each row $j$ for $j \neq p_{1}$ is replaced by that row minus the multiplier $A_{j 1} / A_{p_{1} 1}$ times row $p$. The resulting matrix $\widetilde{A}$, say, is then such that $\widetilde{A}_{j 1}=0, j \neq p_{1}$. The usual practice is then to interchange the pivot row with the first row, by means of a simple permutation, and apply the same procedure to the submatrix $A_{1}=\widetilde{A}_{2 \leq i, 2 \leq j}$ etc.. If we set $A_{0}$ then the matrices $A_{j} \in \mathbb{C}^{k-j, m-j}$ and the process stops after $m-1$ steps.

The elements $\operatorname{Piv}_{j}=\left(A_{j-1}\right)_{p_{j} 1}$ are called the pivot elements and end up being the diagonal entries $U_{j j}$ of the upper triangle matrix $U$. It is then easy to see that (see e.g. [22, Ch.3])

$$
\prod_{t=1}^{s} \operatorname{Piv}_{t}=\operatorname{det}\left((P A)_{1 \leq i \leq s, 1 \leq j \leq s}\right)
$$

where $P$ is the matrix of the permutations used in the process. This determinant formula allows us to interpret the pivot selection process as follows. At any stage the previous pivots have already been chosen and are fixed. Hence maximizing over the candidates in a column to find $\left|\operatorname{Piv}_{s}\right|$ is the same as maximizing $\left|\prod_{t=1}^{s} \operatorname{Piv}_{t}\right|$ and this in turn is equivalent to maximizng over the various subdeterminants $\left|\operatorname{det}\left((P A)_{1 \leq i \leq s, 1 \leq j \leq s}\right)\right|$. Here, the candidate rows each result in a candidate permutation and so, in this formulation, we maximize over the various allowed permutation matrices $P$.

\subsection{Discrete Leja points by LU with partial pivoting.}

Assume now that our compact set $X$ is finite and ordered in some manner. We take

$$
\boldsymbol{x}=X=\left\{x_{1}, \ldots, x_{M}\right\}, A=V(\boldsymbol{x} ; \boldsymbol{p}) \in \mathbb{C}^{M \times N}
$$

where, as before, $N=\operatorname{dim}\left(\mathbb{P}_{n}^{d}(X)\right)$. Our assumption that $X$ is determining implies that $M \geq N$ (indeed typically $M \gg N$ ). We emphasize again 
that the rows of the Vandermonde matrix correspond to the points, and the columns to the basis. Hence selecting rows of $A$ corresponds exactly to selecting points of $X$. Which rows, and hence points of $X$, does partial pivoting select?

By (17) (and the remarks following) the criteria of partial pivoting is to choose the maximum such determinant, in absolute value. Suppose that the rows selected, in sequence, correspond to the points $\xi_{1}, \xi_{2}, \ldots$ etc., then the determinant $\operatorname{det}\left((P A)_{1 \leq i \leq s, 1 \leq j \leq s}\right)$ is just the determinant

$$
v d m\left(\xi_{1}, \ldots, \xi_{s} ; p_{1}, \ldots, p_{s}\right)
$$

and hence partial pivoting chooses the rows corresponding to the Leja points for $X$, in the same order!

In view of the considerations above, we can write the following matrix implementation of the greedy algorithm that computes the Leja points of a WAM, say $X=\mathcal{A}_{n}$, of a polynomial determining compact set $K$ (cf. (11)-(12)). Again, we use a Matlab-like notation:

\section{Algorithm DLP: Discrete Leja Points}

- $\boldsymbol{a}=\mathcal{A}_{n}=\left\{a_{1}, \ldots, a_{M}\right\}$;

- $A=V(\boldsymbol{a} ; \boldsymbol{p})$;

- $[L, U, \boldsymbol{\sigma}]=L U(A$, 'vector' $) ;(\boldsymbol{\sigma}$ is a permutation vector $)$

- ind $=\boldsymbol{\sigma}(1: N) ; \boldsymbol{\xi}=\boldsymbol{a}($ ind $) ;($ extraction of Discrete Leja Points)

When $V(\boldsymbol{a} ; \boldsymbol{p})$ is very ill-conditioned, as typically happens with nonorthogonal bases, we can still apply step $(i)$ of algorithm AFP to get a discrete orthonormal basis and a better conditioned Vandermonde matrix.

Remark 6.1 Observe that the first $k$ Leja points depend only on the first $k$ basis functions in $\boldsymbol{p}$. Hence the Leja points are a sequence, i.e., computing the Leja points for the basis obtained from $\boldsymbol{p}$ by adding an element to the end of $\boldsymbol{p}$ does not change the Leja points already computed for $\boldsymbol{p}$.

In particular, if the basis is chosen so that the first $N_{j}=\operatorname{dim}\left(\mathbb{P}_{j}^{d}(K)\right)$ basis elements span $\mathbb{P}_{j}^{d}(K)$, then the first $N_{j}$ Leja points are a candidate set of interpolation points for polynomials of degree $j$. Moreover, by the nature of the Gram-Schmidt process, this latter property is not affected by a preliminary orthogolization process as in step $(i)$ of algorithm AFP (since the matrix $T_{s}$ is upper triangular).

In contrast, the Approximate Fekete points depend on the entire basis, and not on its order. 


\subsection{Asymptotics of Discrete Leja Points.}

We showed in [8] that the Approximate Fekete Points selected from a WAM $\mathcal{A}_{n} \subset K$, using any polynomial basis, and the true Fekete points for $K$ both have the same asymptotic distribution.

We first introduce some notation. By

$$
\operatorname{vdm}\left(z_{1}, \ldots, z_{N}\right)
$$

we will mean the Vandermonde determinant computed using the standard monomial basis. Further, we set $m_{n}$ to be the sum of the degrees of the $N$ monomials of degree at most $n$, i.e., $m_{n}=d n N /(d+1)$.

Then it is known (see [38]) that if $F_{n}=\left\{f_{1}, \ldots, f_{N}\right\} \subset K$ is a set of true Fekete points for degree $n$ in $K$, the limit

$$
\lim _{n \rightarrow \infty}\left|\operatorname{vdm}\left(f_{1}, \ldots, f_{N}\right)\right|^{1 / m_{n}}=: \tau(K)
$$

exists and is called the transfinite diameter of $K$.

Theorem 6.1 (cf. [8]) Suppose that $K \subset \mathbb{C}^{d}$ is compact, non-pluripolar, polynomially convex and regular (in the sense of Pluripotential theory) and that for $n=1,2, \ldots, \mathcal{A}_{n} \subset K$ is a WAM. Let $\left\{\xi_{1}, \ldots, \xi_{N}\right\}$ be the Approximate Fekete Points selected from $\mathcal{A}_{n}$ by the greedy algorithm AFP described above, using any polynomial basis $\boldsymbol{p}=\left\{p_{1}, \ldots, p_{N}\right\}$. Then

- $\lim _{n \rightarrow \infty}\left|\operatorname{vdm}\left(\xi_{1}, \ldots, \xi_{N}\right)\right|^{1 / m_{n}}=\tau(K)$, the transfinite diameter of $K$;

- the discrete probability measures $\mu_{n}:=\frac{1}{N} \sum_{j=1}^{N} \delta_{\xi_{j}}$ converge weak-* to the pluripotential-theoretic equilibrium measure $d \mu_{K}$ of $K$.

Remark 6.2 For $K=[-1,1], d \mu_{[-1,1]}=\frac{1}{\pi} \frac{1}{\sqrt{1-x^{2}}} d x$; for $K$ the unit circle $S^{1}, d \mu_{S^{1}}=\frac{1}{2 \pi} d \theta$. If $K \subset \mathbb{R}^{d} \subset \mathbb{C}^{d}$ is compact, then $K$ is automatically polynomially convex. We refer the reader to [24] for other examples and more on complex pluripotential theory.

Remark 6.3 Note also that a set of true Fekete points $F_{n}$ is also a WAM and hence we may take $\mathcal{A}_{n}=F_{n}$, in which case the algorithm will select as Approximate Fekete Points exactly $F_{n}$ (there is no other choice) and so the true Fekete points must necessarily also have these two properties.

Leja points depend more strongly on the ordering of the basis than do Fekete points and hence it is perhaps not surprising that the convergence theorem for Leja points that we are able to offer is slightly weaker than that for Fekete points. In fact, we give a convergence theorem that holds for a certain class of ordered bases. Specifically, let

$$
e=\left\{e_{1}, \ldots, e_{N}\right\}
$$


be any ordering of the standard monomials $z^{\alpha}$ that is consistent with the degree, i.e.

$$
j \leq k \Longrightarrow \operatorname{deg}\left(e_{j}\right) \leq \operatorname{deg}\left(e_{k}\right) .
$$

We consider the ordered bases of $\mathbb{P}_{n}^{d}, \boldsymbol{p}=\left\{p_{1}, \ldots, p_{N}\right\}$, that can be written in the form

$$
\boldsymbol{p}=L \boldsymbol{e}
$$

where $L \in \mathbb{C}^{N \times N}$ is lower triangular. Note that these are exactly the kind of bases of which we discussed in Remark 6.1.

Theorem 6.2 Suppose that $K \subset \mathbb{C}^{d}$ is compact, non-pluripolar, polynomially convex and regular (in the sense of Pluripotential theory) and that for $n=1,2, \cdots, \mathcal{A}_{n} \subset K$ is a WAM. Let $\left\{\xi_{1}, \ldots, \xi_{N}\right\}$ be the Discrete Leja Points selected from $\mathcal{A}_{n}$ by the greedy algorithm DLP described above, using any basis $\boldsymbol{p}$ of the form (18). Then

- $\lim _{n \rightarrow \infty}\left|\operatorname{vdm}\left(\xi_{1}, \ldots, \xi_{N}\right)\right|^{1 / m_{n}}=\tau(K)$, the transfinite diameter of $K$;

- the discrete probability measures $\mu_{n}:=\frac{1}{N} \sum_{j=1}^{N} \delta_{\xi_{j}}$ converge weak-* to the pluripotential-theoretic equilibrium measure $d \mu_{K}$ of $K$.

Proof. The proof of the first assertion is based on a slight modification of the proof of Proposition 3.7 of [5], which concerns the true Leja points of $K$. Once we have established the first, then the second assertion follows, as in the proof of [8, Thm.1], from the main result of [3].

First note that, since the $k$-th column of $v d m\left(\mathcal{A}_{n} ; \boldsymbol{p}\right)$ consists of $p_{k}$ evaluated at all the points of $\mathcal{A}_{n}$. Further, since the pivoting strategy is a comparison of elements in a column, the order of rows selected is not affected by multiplying a column, i.e., multiplying a $p_{k}$, by a constant. Hence, without loss of generality, we may assume that the diagonal elements $L_{j j}$ of the transformation in (18) are $L_{j j}=1$. Hence each $p_{k}$ may be assumed to be of the form

$$
p_{k}(z)=e_{k}(z)+\sum_{j<k} c_{j} e_{j}(z), \quad c_{j} \in \mathbb{C} .
$$

Moreover, each combination

$$
p_{k}(z)+\sum_{j<k} a_{j} p_{j}(z)=e_{k}(z)+\sum_{j<k} c_{j} e_{j}(z)
$$

is of exactly the same form.

It follows that the so-called Chebyshev constants

$$
t_{k}(K):=\inf _{c_{j} \in \mathbb{C}}\left\|e_{k}(\cdot)+\sum_{j<k} c_{j} e_{j}(\cdot)\right\|_{K}^{1 / \operatorname{deg}\left(e_{k}\right)}
$$


may also be defined as

$$
t_{k}(K):=\inf _{c_{j} \in \mathbb{C}}\left\|p_{k}(\cdot)+\sum_{j<k} c_{j} p_{j}(\cdot)\right\|_{K}^{1 / \operatorname{deg}\left(p_{k}\right)}
$$

and similarly for the Chebyshev constants for $\mathcal{A}_{n}, t_{k}\left(\mathcal{A}_{n}\right)$.

We also need to make use of the geometric mean of the Chebyshev constants, i.e.,

$$
t_{k}^{0}(K):=\left(\prod_{\operatorname{deg}\left(e_{j}\right)=k} t_{j}(K)\right)^{1 /\left(N_{k}-N_{k-1}\right)}
$$

where $N_{k}:=\operatorname{dim}\left(\mathbb{P}_{k}^{d}(K)\right)$.

Note also, that in our case, $\mathcal{A}_{n}$ is assumed to be a WAM and hence

$$
\left(t_{k}\left(\mathcal{A}_{n}\right)\right)^{\operatorname{deg}\left(e_{k}\right)} \geq \frac{1}{C\left(\mathcal{A}_{n}\right)}\left(t_{k}(K)\right)^{\operatorname{deg}\left(e_{k}\right)}, \quad 1 \leq k \leq N_{n} .
$$

Consequently we also have

$$
t_{k}^{0}\left(\mathcal{A}_{n}\right) \geq\left(\frac{1}{C\left(\mathcal{A}_{n}\right)}\right)^{1 / r_{k}} t_{k}^{0}(K)
$$

where

$$
r_{k}:=k\left(N_{k}-N_{k-1}\right)
$$

Thus, by (3.7.3) of [5] we have

$$
\left|\operatorname{vdm}\left(\xi_{1}, \ldots, \xi_{N}\right)\right| \geq\left(\frac{1}{C\left(\mathcal{A}_{n}\right)}\right)^{N_{n}} \prod_{k=1}^{n}\left(t_{k}^{0}(K)\right)^{r_{k}} .
$$

By the definition of Fekete points we even have

$$
\left|\operatorname{vdm}\left(f_{1}, \ldots, f_{N}\right)\right| \geq\left|\operatorname{vdm}\left(\xi_{1}, \ldots, \xi_{N}\right)\right| \geq\left(\frac{1}{C\left(\mathcal{A}_{n}\right)}\right)^{N_{n}} \prod_{k=1}^{n}\left(t_{k}^{0}(K)\right)^{r_{k}}
$$

Note then that, by the definition of $C\left(\mathcal{A}_{n}\right),\left(\frac{1}{C\left(\mathcal{A}_{n}\right)}\right)^{N_{n} / m_{n}} \rightarrow 1$ and

$$
\left(\prod_{k=1}^{n}\left(t_{k}^{0}(K)\right)^{r_{k}}\right)^{1 / m_{n}}=\tau(K)
$$

by the famous result of Zaharjuta [38]. The result follows.

Remark 6.4 The idea of computing Leja sequences from Weakly Admissible Meshes appears also in the recent paper [4], which concerns the univariate complex case. The approach is there embedded in the more general concept of Pseudo Leja Sequence, for which univariate versions of the asymptotic results above have been proved. The present LU-based strategy can be viewed as an alternative computational method for producing such "discrete" univariate Leja sequences. 


\section{Numerical examples.}

In this section we present some examples of computation of Discrete Leja Points (DLP) and Approximate Fekete Points (AFP) by the matrix algorithms discussed in Sections 5 and 6.

\subsection{A circular sector.}

As a first example, we take the circular sector (3/4 of the unit disk, written in polar coordinates)

$$
K=\{(\rho, \theta): 0 \leq \rho \leq 1,-\pi / 2 \leq \theta \leq \pi\}
$$

which has been already considered in [32, Ex.2]. Our first step is to construct an Admissible Mesh (AM) for $K$, that will be used to extract DLP and AFP. To this purpose, in view of Property $\mathrm{P} 4$, it is convenient to see $K$ as the union of three quadrants, since these are convex compact sets where we can easily compute an AM.

We show now, following the proof of [15, Thm.5] (which is much more general), how to construct an $\mathrm{AM}$ in a convex compact set $K \subset \mathbb{R}^{2}$. First, we recall that every convex compact set of $\mathbb{R}^{2}$ admits the Markov inequality

$$
\max _{x \in K}\|\nabla p(x)\|_{2} \leq M n^{2}\|p\|_{K}, \quad M=\frac{\alpha(K)}{w(K)}, \quad \forall p \in \mathbb{P}_{n}^{2}(K),
$$

where $\alpha(K) \leq 4$, and $w(K)$ is the minimal distance between two parallel supporting lines for $K$, cf. [26].

Consider a cartesian grid $\{(i h, j h), i, j \in \mathbb{Z}\}$ with constant stepsize $h$ : for every square of the grid that has nonempty intersection with $K$, take a point in this intersection. Let $\mathcal{A}_{n}$ be the mesh formed by such points. For every $x \in K$, let $a \in \mathcal{A}_{n}$ the point closest to $x$ : by construction, the distance between these points cannot exceed $\sqrt{2} h$. Using the mean value theorem, the Cauchy-Schwarz inequality and the Markov inequality, we can write

$$
|p(x)-p(a)| \leq\|\nabla p(y)\|_{2}\|x-a\|_{2} \leq M \sqrt{2} h n^{2}\|p\|_{K},
$$

since $y$ belongs to the open segment connecting $x$ and $a$, which lies in $K$. Then, from $|p(x)| \leq|p(x)-p(a)|+|p(a)| \leq M \sqrt{2} h n^{2}\|p\|_{K}+|p(a)|$, the polynomial inequality

$$
\|p\|_{K} \leq \frac{1}{1-\mu}\|p\|_{\mathcal{A}_{n}}
$$

follows, provided that

$$
h=h_{n}: M \sqrt{2} h_{n} n^{2} \leq \mu<1,
$$

i.e., $\mathcal{A}_{n}$ is an $\mathrm{AM}$ with constant $C=1 /(1-\mu)$, cf. (10). 
In the case of the first quadrant of the unit disk, we have that $w(K)=1$, and we take the upper bound for convex compacts $\alpha(K)=4$, since sharper bounds do not seem to be available in the literature. Hence, by (22)-(23) we get an $\mathrm{AM}$ as soon as we consider the cartesian grid $\left\{\left(i h_{n}, j h_{n}\right), i, j \in \mathbb{Z}\right\}$ with $4 \sqrt{2} n^{2} h_{n}<5.66 n^{2} h_{n} \leq \mu<1$ for some fixed $\mu$. For example, taking $h_{n}=\left(6 n^{2}\right)^{-1}$ we get $\mu=5.66 / 6$ and $C \approx 17.65$. Since we can partition the set of grid squares into subsets of four adjacent squares and, apart from a neighborhood of the boundary of the quadrant, take as mesh point their common vertex, then the cardinality of the mesh is roughly estimated as $1 / 4$ of the number of grid points in the unit square times the area of the quadrant, that is $\operatorname{card}\left(\mathcal{A}_{n}\right) \approx\left(\left(6 n^{2}\right)^{2} / 4\right) \pi / 4 \approx 7 n^{4}$. We could obtain a lower constant $C$, paying the price of increasing the cardinality of the mesh.

By Property P1 and P4 of admissible meshes then we get an AM of the compact set $(21)$ as union of three meshes of the three quadrants, with the same constant $C \approx 17.65$ and cardinality $\operatorname{card}\left(\mathcal{A}_{n}\right) \approx 3 \times 7 n^{4}=21 n^{4}$. In Figure 1 we show the DLP and AFP computed for degree $n=6$, with the Koornwinder orthogonal basis of the unit disk for the Vandermonde matrix (cf. [25]). In this example the Vandermonde matrix is not ill-conditioned, so the preliminary orthogonalization iterations are not necessary. It is worth noticing two facts. The first is that in our implementation (Matlab 7.6.0 on an Intel Core 2 Duo 2.13GHz Processor with 4Gb RAM), the computation of DLP is 3 times faster than that of AFP (around $0.03 \mathrm{~s}$ versus $0.09 \mathrm{~s}$ ). On the other hand, the quality of AFP is better than that of DLP. Not only do they appear more evenly distributed, but in addition the absolute value of the Vandermonde determinant and the Lebesgue constant (numerically evaluated) are $|v d m| \approx 2 \cdot 10^{4}$ and $\Lambda_{6} \approx 4$ for the AFP, whereas $|v d m| \approx 7 \cdot 10^{2}$ and $\Lambda_{6} \approx 12$ for the DLP. Notice that both the Lebesgue constants are much below the theoretical bound for Fekete points extracted from an AM, namely $\Lambda_{n} \leq C N$.

In this example we are already using for a low degree a huge number of mesh points, namely around 27000 points. This is a typical situation with Admissible Meshes, since their cardinality increases like $\mathcal{O}\left(n^{4}\right)$. It is therefore necessary to reduce the cardinality of the extraction meshes, even for low/moderate degrees. This can be obtained using Weakly Admissible Meshes, when available, as is done in the next subsection with triangles and polygons.

\subsection{Polygons.}

The recent paper [11] gives a construction of a WAM for the two-dimensional unit simplex, and thus for any triangle by affine mapping (Property P1 of WAMs). This WAM, say $\mathcal{A}_{n}$, has $n^{2}+n+1$ points for degree $n$, and constant $C\left(\mathcal{A}_{n}\right)=\mathcal{O}\left(\log ^{2} n\right)$. The mesh points lie on a grid of intersecting straight lines, namely a pencil from one vertex (image of the point $(0,0)$ 


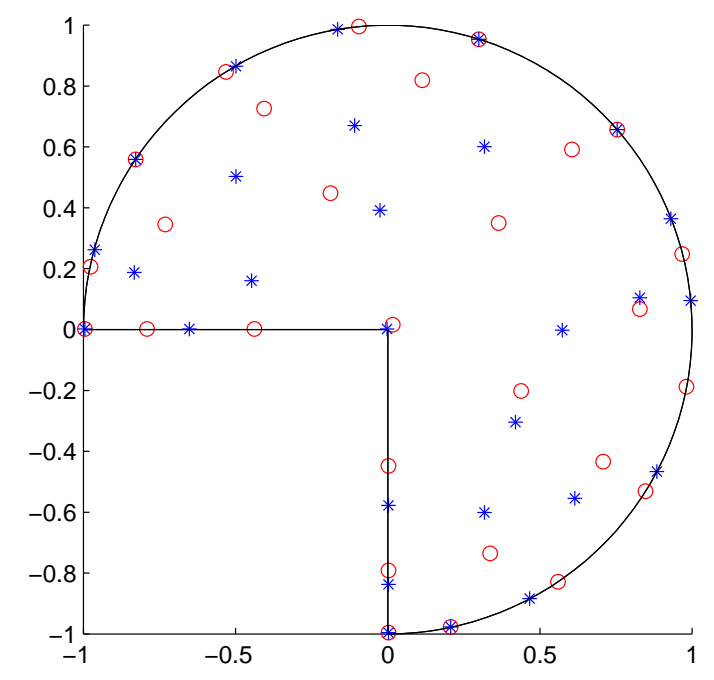

Figure 1: $N=28$ AFP (Approximate Fekete Points, circles) and DLP (Discrete Leja Points, asterisks) for degree $n=6$ extracted from an AM (Admissible Mesh) of a circular sector.

of the simplex) cut by a pencil parallel to the opposite side (image of the hypothenuse of the simplex). The points on each segment of the pencils, and in particular the points on each side, are the corresponding ChebyshevLobatto points.

Property P4 allows then to obtain WAMs for any triangulated polygon. The constant of any such WAM can be bounded by the maximum of the constants corresponding to the triangular elements, and thus is $\mathcal{O}\left(\log ^{2} n\right)$, irrespectively of the number of sides of the polygon, or of the fact that it is convex or concave. Notice that a rough triangulation is better in the present framework, to keep the cardinality of the mesh low (which will be of the order of $n^{2}$ times the number of triangles).

As a first example, we consider a nonregular convex hexagon, either trivially triangulated by the barycenter, or by the so-called "ear-clipping" algorithm (cf., e.g., [20]). The latter constructs a minimal triangulation of any simple polygon with $k$ vertices, obtaining $k-2$ triangles. In Figure 2, we show the $N=45 \mathrm{AFP}$ and DLP computed for degree $n=8$, using the product Chebyshev basis of the minimal surrounding rectangle for the Vandermonde matrix. In the first mesh the point $(0,0)$ of the simplex is mapped to the barycenter for each triangle. The cardinality of the barycentric-based mesh is $6\left(n^{2}+n+1\right)-6(n+1)-5=6 n^{2}-5$, whereas that of the other mesh is $4\left(n^{2}+n+1\right)-3(n+1)-2=4 n^{2}+n-1$ (one has to subtract the repetitions of points along the contact sides). 

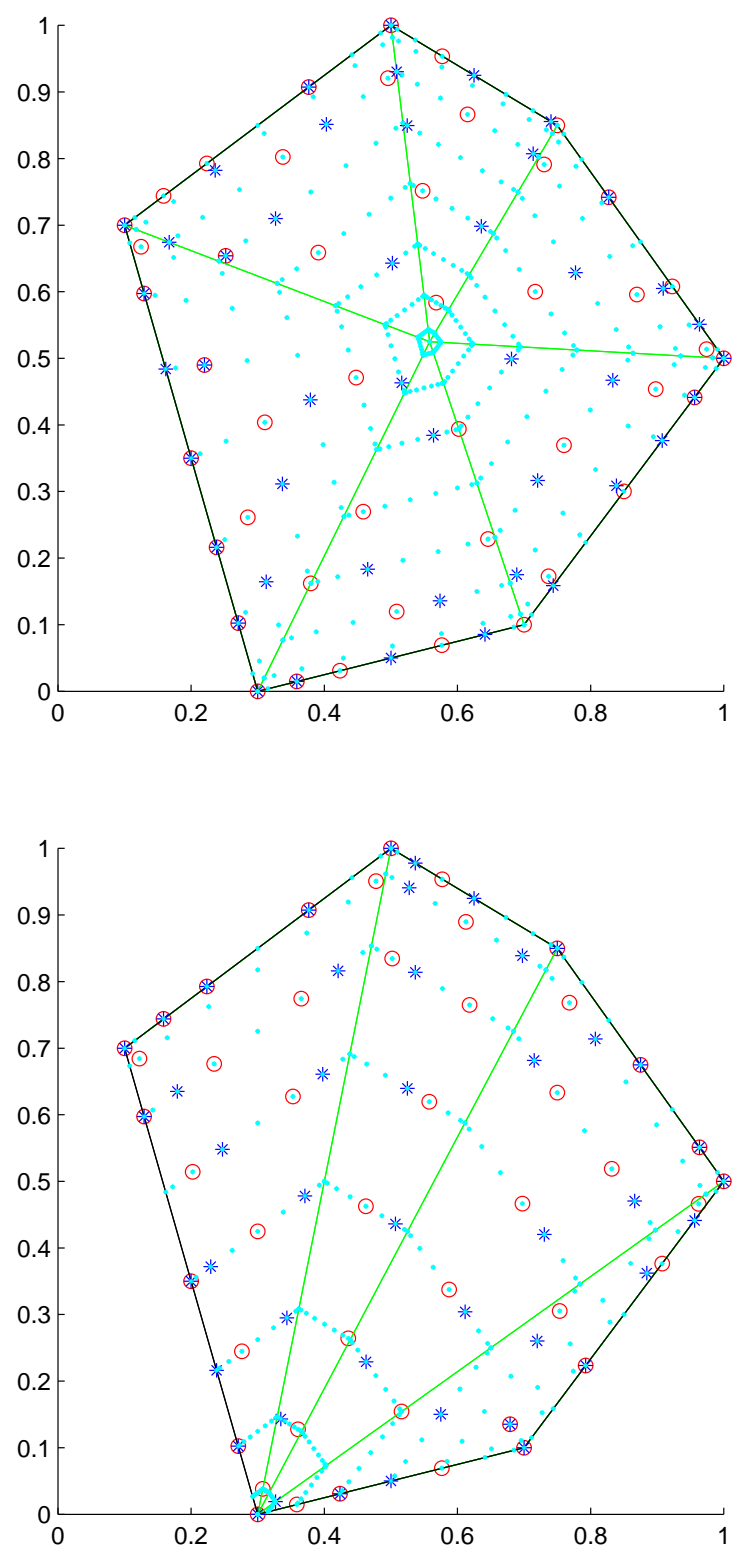

Figure 2: $N=45$ AFP (circles) and DLP (asterisks) for degree $n=8$ extracted from two WAMs of a nonregular convex hexagon (dots).

In Table 1 we show the numerically evaluated Lebesgue constants for the AFP and DLP computed from the two meshes above, at a sequence of degrees. From these results we see that DLP are of lower quality than AFP: this is not surprising, since the same phenomenon is well-known concerning 
continuous Fekete and Leja points. Nevertheless, both provide reasonably good interpolation points, as it is seen from the interpolation errors on three test functions in Table 2 .

In order to emphasize the flexibility of Algorithms AFP and DLP, finally we show the points computed for a more complicated polygon in a shape of a hand, with 39 sides (obtained from the screen sampled hand of one of the authors, by piecewise linear interpolation); see Figure 3. In this example we have used the ear-clipping triangulation which gives 37 triangles and a WAM with approximately $37 n^{2}$ points for degree $n$.

Table 1: Lebesgue constants for AFP and DLP extracted from two WAMs of a nonregular convex hexagon (WAM1 corresponding to barycentric triangulation, WAM2 to minimal triangulation).

\begin{tabular}{cccccccc} 
mesh & points & $n=5$ & $n=10$ & $n=15$ & $n=20$ & $n=25$ & $n=30$ \\
\hline WAM1 & AFP & 6.5 & 18.9 & 20.4 & 40.8 & 73.3 & 73.0 \\
& DLP & 7.1 & 19.6 & 49.8 & 58.3 & 108.0 & 167.0 \\
\hline WAM2 & AFP & 6.8 & 12.3 & 34.2 & 52.3 & 49.0 & 80.4 \\
& DLP & 10.7 & 48.4 & 62.0 & 91.6 & 86.6 & 203.0 \\
\hline
\end{tabular}

Table 2: Max-norm of the interpolation errors with AFP and DLP extracted from WAM2 for three test functions: $f_{1}=\cos \left(x_{1}+x_{2}\right) ; f_{2}=$ Franke function; $f_{3}=\left(\left(x_{1}-0.5\right)^{2}+\left(x_{2}-0.5\right)^{2}\right)^{3 / 2}$.

\begin{tabular}{cccccccc} 
function & points & $n=5$ & $n=10$ & $n=15$ & $n=20$ & $n=25$ & $n=30$ \\
\hline$f_{1}$ & AFP & $6 \mathrm{E}-06$ & $5 \mathrm{E}-13$ & $3 \mathrm{E}-15$ & $3 \mathrm{E}-15$ & $3 \mathrm{E}-15$ & $4 \mathrm{E}-15$ \\
& DLP & $8 \mathrm{E}-06$ & $2 \mathrm{E}-12$ & $2 \mathrm{E}-15$ & $4 \mathrm{E}-15$ & $3 \mathrm{E}-15$ & $4 \mathrm{E}-15$ \\
\hline$f_{2}$ & AFP & $1 \mathrm{E}-01$ & $2 \mathrm{E}-02$ & $5 \mathrm{E}-03$ & $4 \mathrm{E}-04$ & $3 \mathrm{E}-05$ & $2 \mathrm{E}-06$ \\
& DLP & $3 \mathrm{E}-01$ & $2 \mathrm{E}-02$ & $9 \mathrm{E}-03$ & $5 \mathrm{E}-04$ & $4 \mathrm{E}-05$ & $3 \mathrm{E}-06$ \\
\hline$f_{3}$ & AFP & $3 \mathrm{E}-03$ & $2 \mathrm{E}-04$ & $1 \mathrm{E}-04$ & $4 \mathrm{E}-05$ & $2 \mathrm{E}-05$ & $1 \mathrm{E}-05$ \\
& DLP & $3 \mathrm{E}-03$ & $3 \mathrm{E}-04$ & $1 \mathrm{E}-04$ & $3 \mathrm{E}-05$ & $2 \mathrm{E}-05$ & $5 \mathrm{E}-06$ \\
\hline
\end{tabular}

Acknowledgements. We are grateful to Robert Schaback for having pointed out to us the LU-based strategy for computing discrete Leja-like points. 


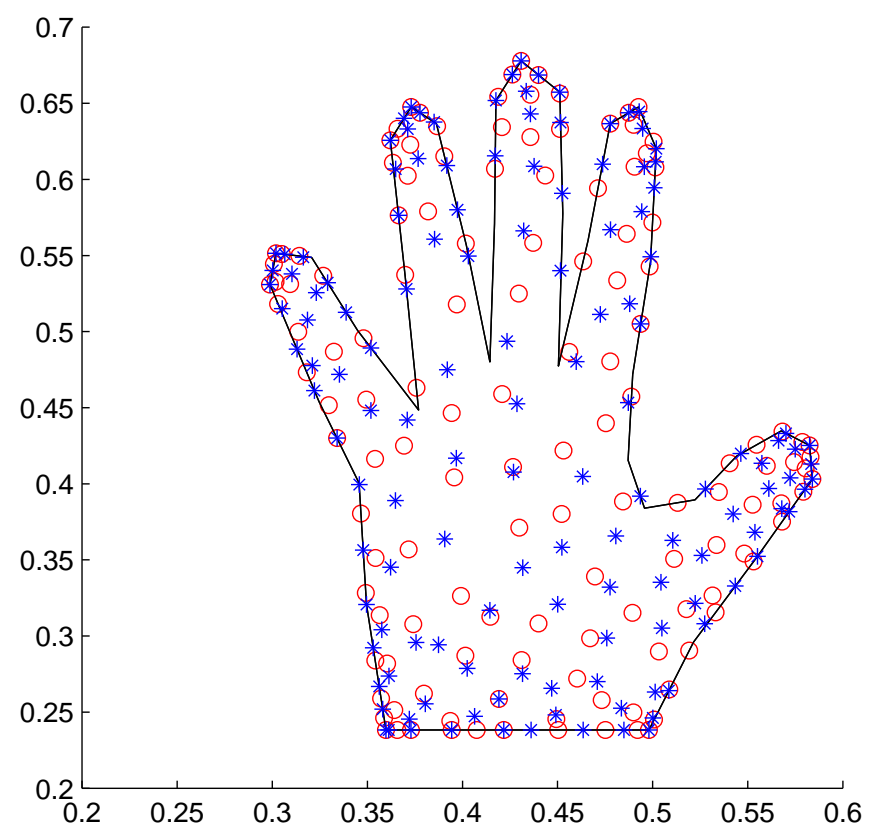

Figure 3: $N=136$ AFP (circles) and DLP (asterisks) for degree $n=15$ in a hand-shape polygon.

\section{References}

[1] J. Baglama, D. Calvetti and L. Reichel, Fast Leja points, Electron. Trans. Numer. Anal. 7 (1998), 124-140.

[2] E. Bendito, A. Carmona, A.M. Encinas and J.M. Gesto, Estimation of Fekete points, J. Comput. Phys. 225 (2007), 2354-2376.

[3] R. Berman and S. Boucksom, Equidistribution of Fekete Points on Complex Manifolds, preprint (online at: http://arxiv.org/abs/0807.0035).

[4] L. Bialas-Ciez and J.-P. Calvi, Pseudo Leja Sequences, draft, February 2009 .

[5] T. Bloom, L. Bos, C. Christensen and N. Levenberg, Polynomial interpolation of holomorphic functions in $C$ and $C^{n}$, Rocky Mountain J. Math. 22 (1992), 441-470.

[6] T. Bloom, L. Bos, N. Levenberg and S. Waldron, On the Convergence of Optimal Measures, Constr. Approx., to appear. 
[7] L. Bos, M. Caliari, S. De Marchi, M. Vianello and Y. Xu, Bivariate Lagrange interpolation at the Padua points: the generating curve approach, J. Approx. Theory 143 (2006), 15-25.

[8] L. Bos, J.-P. Calvi, N. Levenberg, A. Sommariva and M. Vianello, Geometric Weakly Admissible Meshes, Discrete Least Squares Approximation and Approximate Fekete Points, Math. Comp., to appear.

[9] L. Bos and N. Levenberg, On the Approximate Calculation of Fekete Points: the Univariate Case, Electron. Trans. Numer. Anal. 30 (2008), 377-397.

[10] L. Bos, N. Levenberg and S. Waldron, On the Spacing of Fekete Points for a Sphere, Ball or Simplex, Indag. Math. 19 (2008), 163-176.

[11] L. Bos, A. Sommariva and M. Vianello, Least-squares polynomial approximation on weakly admissible meshes: disk and triangle, J. Comput Appl. Math., published online 30 June 2010.

[12] L. Bos, M.A. Taylor and B.A. Wingate, Tensor product Gauss-Lobatto points are Fekete points for the cube, Math. Comp. 70 (2001), 15431547 .

[13] P.A. Businger and G.H. Golub, Linear least-squares solutions by Householder transformations, Numer. Math. 7 (1965), 269-276.

[14] M. Caliari, S. De Marchi and M. Vianello, Bivariate polynomial interpolation at new nodal sets, Appl. Math. Comput. 165 (2005), 261-274.

[15] J.P. Calvi and N. Levenberg, Uniform approximation by discrete least squares polynomials, J. Approx. Theory 152 (2008), 82-100.

[16] A. Civril and M. Magdon-Ismail, On Selecting a Maximum Volume Sub-matrix of a Matrix and Related Problems, Theoretical Computer Science 410 (2009), 4801-4811.

[17] S. De Marchi, On Leja sequences: some results and applications, Appl. Math. Comput. 152 (2004), 621-647.

[18] M. Dubiner, Spectral methods on triangles and other domains, J. Sci. Comput. 6 (1991), 345-390.

[19] C.F. Dunkl and Y. Xu, Orthogonal polynomials of several variables, Cambridge University Press, Cambridge, 2001.

[20] A. Fournier and D.Y. Montuno, Triangulating simple polygons and equivalent problems, ACM Trans. on Graphics 3 (1984), 153-174. 
[21] L. Giraud, J. Langou, M. Rozloznik and J. van den Eshof, Rounding error analysis of the classical Gram-Schmidt orthogonalization process, Numer. Math. 101 (2005), 87-100.

[22] G.H. Golub and C.F. Van Loan, Matrix computations, third edition, Johns Hopkins University Press, Baltimore, MD, 1996.

[23] M. Götz, On the distribution of Leja-Górski points, J. Comput. Anal. Appl. 3 (2001), 223-241.

[24] M. Klimek, Pluripotential Theory, Oxford U. Press, 1991.

[25] T. Koornwinder, Two-variable analogues of the classical orthogonal polynomials, Theory and application of special functions (Proc. Advanced Sem., Math. Res. Center, Univ. Wisconsin, Madison, Wis., 1975), 435-495.

[26] A. Kroó and S. Révész, On Bernstein and Markov-type Inequalities for Multivariate Polynomials on Convex Bodies, J. Approx. Theory 99 (1999), 134-152.

[27] E. Levin and E.B. Saff, Potential theoretic tools in polynomial and rational approximation, in: Harmonic analysis and rational approximation, 71-94, Lecture Notes in Control and Inform. Sci., 327, Springer, Berlin, 2006.

[28] A. Lopez and E. Saff, Asymptotics of Greedy Energy Points, Math. Comp., to appear.

[29] The Mathworks, MATLAB documentation set, 2009 version (online at: http://www.mathworks.com).

[30] L. Reichel, Newton interpolation at Leja points, BIT 30 (1990), 332346.

[31] E.B. Saff and V. Totik, Logarithmic potentials with external fields, Springer, Berlin, 1997.

[32] R. Schaback and S. De Marchi, Nonstandard Kernels and Their Applications, Dolomites Research Notes on Approximation (DRNA) 2 (2009), 16-43.

[33] I.H. Sloan and R.S. Womersley, Extremal systems of points and numerical integration on the sphere, Adv. Comput. Math. 21 (2004), 107-125.

[34] A. Sommariva and M. Vianello, Computing approximate Fekete points by QR factorizations of Vandermonde matrices, Comput. Math. Appl. 57 (2009), 1324-1336. 
[35] A. Sommariva and M. Vianello, Approximate Fekete points for weighted polynomial interpolation, Electron. Trans. Numer. Anal. 37 (2010), 122.

[36] M.A. Taylor, B.A. Wingate and R.E. Vincent, An algorithm for computing Fekete points in the triangle, SIAM J. Numer. Anal. 38 (2000), 1707-1720.

[37] T. Warburton, An explicit construction of interpolation nodes on the simplex, J. Engrg. Math. 56 (2006), 247-262.

[38] V.P. Zaharjuta, Transfinite diameter, Chebyshev constants and capacity for compacts in $\mathbb{C}^{n}$, Math. USSR Sbornik 25 (1975), 350-364. 